\title{
Comparison of Reactive Routing Protocols in MANETs
}

\author{
Jagpal S. Ubhi and Satveer Kaur
}

\begin{abstract}
Mobile wireless Ad Hoc networks (MANET) is an infrastructure free network, where every node functions as transmitter, router and data sink. Mobility and node density are the fundamental characteristics which differentiate MANET from other wireless or wired networks. Therefore, MANET routing protocols are designed to adaptively cater to dynamic changes in topology while maximizing the throughput and packet delivery ratio, and minimizing delay, aggregate good put, average jitter and minimum packet loss. In this paper, Ad Hoc Demand Vector (AODV) and Dynamic Source Routing (DSR) are used to implement the MANET which have been simulated on QualNet5.0 simulator. The impact on mobility and change in node density factor in MANET is investigated and compared for reactive routing protocols such as AODV and DSR. The simulative study on MANET routing protocols aims to determine the performance of current MANET routing protocols with respect to mobility and node density factors. Results vary when we change the node density. It is observed that an increase in node density has different impact on all routing protocols under various mobility patterns, i.e. a degradation of the network performance. However, the degree of degradation varies for different combinations of protocols. The performance of RWP model provides a baseline to judge the quality of routing protocols when there is no group movement. The results of this network are tabulated along with a comprehensive analysis which compares throughput, packet delivery ratio, end to end delay, aggregate good put, average jitter value and packet dropping.
\end{abstract}

Index Terms-MANET, QualNet5.0, AODV, DSR.

\section{INTRODUCTION}

The wireless multi-hop networks such as ad hoc networks, sensor networks and vehicular networks have been very extensively used for research purposes. A Mobile Ad Hoc Network (MANET) is a collection of wireless mobile terminals which are able to dynamically form a temporary network without use of any fixed infrastructure and/or without centralized administration. In recent years, MANET is an attraction and attention for potential use in several fields. Even, in case the total number of nodes in the MANET becomes very large, then the overhead of the employed routing algorithms should be low and independent of the total number of nodes in MANET. The absence of any fixed infrastructure make MANET very attractive for rescue operations and time-critical applications. The nodes are free to move randomly, the topology of network may change rapidly and become unpredictable. This makes the traditional protocols not suitable for MANET. The ongoing

Manuscript received June 9, 2015; revised August 8, 2015

Jagpal S. Ubhi is with the Sant Longowal Institute of Engineering \& Technology, Longowal, District Sangrur (Punjab), India (e-mail: js_ubhi@yahoo.com).

Satveer Kaur was with Guru Nanak Dev University Regional Campus, Sathiala, District Amritsar (Punjab), India (e-mail: rattansatveer1985@gmail.com). transmissions is influenced by mobility of nodes as the mobile node that receive and forwards packets may move out of range. Reference [1] The movement pattern of MANET nodes is characterized by mobility models and each routing protocols exhibits specific characteristics of these models. Reference [2] In order to find the most adaptive and efficient routing protocol for dynamic MANET topologies, the behavior of routing protocols needs to be analyzed at varying node speeds, number of traffic nodes, network size, as well as node density. So there is need to evaluate the performance of routing protocols in different mobility scenarios and based on the comparison a protocol for a particular scenario is selected. In this paper, performance comparison of two prominent MANET routing protocols Ad Hoc On Demand Vector (AODV) and Dynamic Source Routing (DSR) is presented by running simulations on QualNet5.0. The performance analysis is restricted to performance metrics.

\section{ClassificAtio of Manet Routing Protocols}

MANET routing protocols are IP based which may use unicast, multicast or hybrid approaches. Reference [3] These may be allowed for interaction with standard wired IP services rather than being regarded as a completely independent or separate entity. Fig. 1 shows the categorization of different routing protocols of MANET.

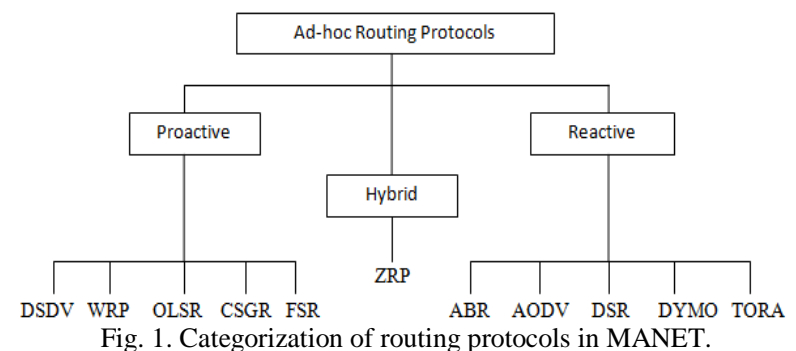

\section{A. Reactive Routing Protocols}

These are also known as Demand based routing protocols in which a route is discovered only when need arises. Nodes maintain routes to active destinations only. The communication overhead is reduced at the expense of delay as a route is to be discovered. Reference [4] In Adhoc environment battery power is conserved both by not sending the advertisements and by not receiving so reactive routing protocols are considered to be very significant. All nodes maintain the record of discovered routes in their routing tables. However, only valid routes are kept and all the other/old routes are deleted after an active route timeout. The problem in MANET arises when there is link failures occur due to higher node mobility and during the same time new links may also be established between previously 
distant nodes which significantly increases the network broadcast traffic with rapid link make/break effect of intermediate nodes. Reference [5] The path discovery process for a reactive routing protocol has been shown in Fig. 2.

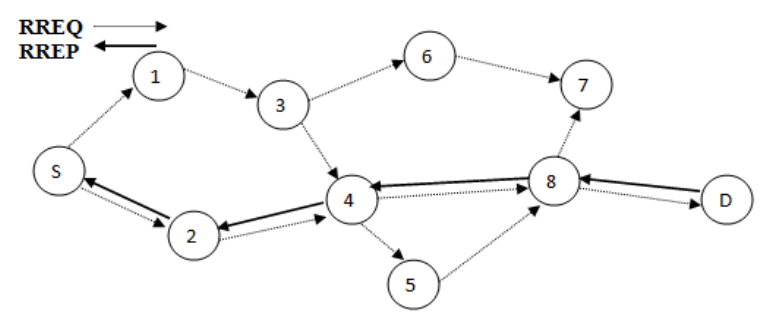

Fig. 2. Discovery process.

In recent years, following are some of the reactive routing protocols used in MANET:

Adhoc on Demand Distance Vector (AODV): Adhoc on Demand Distance Vector is a reactive protocol which only requests a route when it needs one. Reference [6] It does not require that the mobile nodes maintains the routes to destinations which are not communicating. It guarantees loop free routes by using sequence numbers that indicate the freshness of a route. In AODV, routing table keeps a record of one route entry for every node communicating with the destination node. Each route entry keeps track of certain fields like Destination IP Address, Destination sequence number, Next Hop, Hop Count. In AODV, a node broadcasts a route request (RREQ) packet in order to find a path to a destination node. The RREQ contains IP address of the node alongwith current sequence number, broadcast identity and most recent sequence number of the destination node which is known to the source node. On the receipt of RREQ the destination node, unicasts a route reply (RREP) packet along the reverse path established at the intermediate nodes during the route discovery process. In case, there is any link failure, a route error (RERR) packet is sent to the source and destination nodes. Reference [7] The source nodes, with the help of sequence numbers are always able to find new valid routes.

Dynamic Source Routing (DSR): It also uses the source routing strategy to establish a route to the destination when a source node makes a request. In case of source routing, the source must know the complete sequence of hops that each packet should traverse to destination. Each node maintains a route cache, where all routes known by the node, are stored. The route discovery process is initiated in case the desired route is not found in the route cache. To limit the number of route requests propagated, a node processes the route request message only if it has not already received the message and its address is not present in the route record of the message. Source routing requires the sequence of hops included in each packet's header. A negative consequence of this is the routing overhead that every packet has to carry. However, one big advantage is that intermediate nodes can learn routes from the source routes in the packets they receive. Reference [8] Source routing is preferred as finding a route is generally a costly operation otherwise in terms of time, bandwidth and energy. Also it avoids the need for upto-date routing information in the intermediate nodes included in the packets. Reference [9] It also avoids routing loops easily because the complete route is determined by a single node instead of making the decision based on hop-byhop.

\section{B. Proactive Routing Protocols}

These are also known as Table driven approaches routing protocols in which every node continuously maintains the complete routing information of the network. Reference [10] When a node needs to forward a packet, there is no delay in searching for a root as it is readily available. Reference [11] But for highly dynamic topology, the proactive schemes spend a significant amount of scarce wireless resources to keep complete and correct routing information. In case of a link breakage is very frequent then the proactive routing protocols need a higher rate of routing table updates affecting the performance of the network.

\section{Hybrid Routing Protocols}

Reference [12] A hybrid Routing protocols are based on a Distance Vector protocol but contain many of the features and advantages of Link State Routing protocols. e.g. EIGRP (Enhanced Interior Gateway Routing Protocol) and ZRP (Zone Routing Protocol).

\section{RANDOM WAYPOINT MOBILITY MODEL}

In MANET, the movement of nodes is characterized by the rate of change of speed and direction. Random Waypoint Model (RWP) is the most widely used mobility model for simulating different routing protocols. It's a 3-tuple is ( $V_{\max }$, $\left.T, V_{i}\right)$; where the node velocity is uniformly distributed from 0 to $V_{\max }, T$ is the pause time and $V_{i}$ is the direction or advance vector. In RWP model, a node randomly chooses a destination, called waypoint and moves towards it in a straight line with constant velocity from uniformly distributed velocity range. After reaching the waypoint, the node pauses for some time and then repeats the same procedure. Mathematically, for currently node which is at point $d(x-1, y-1)$, the next waypoint is given as:

$$
d(x, y)=d(x-1, y-1)+V_{i}
$$

\section{PERFORMANCE METRICS}

\begin{tabular}{|c|c|c|c|}
\multicolumn{4}{|c}{ TABLE I: SIMULATION PARAMETERS LIST } \\
\hline Sr. & \multicolumn{3}{|c|}{ Parameters List } \\
\cline { 2 - 5 } No. & Experiment Parameter & $\begin{array}{c}\text { Experiment } \\
\text { Value }\end{array}$ & Description \\
\hline 1 & Simulation Time & $399 \mathrm{~s}$ & Simulation Duration \\
\hline 2 & Terrain Dimension & {$[1500 \times 1500] \mathrm{m}$} & $\begin{array}{c}X, Y \text { Dimension of } \\
\text { motion }\end{array}$ \\
\hline 3 & No. of mobile nodes & 50 & $\begin{array}{c}\text { No. of nodes in a } \\
\text { network }\end{array}$ \\
\hline 4 & Node Placement & $\begin{array}{c}\text { Random } \\
\text { Waypoint }\end{array}$ & $\begin{array}{c}\text { Change Direction } \\
\text { randomly }\end{array}$ \\
\hline 5 & Mobility Speed & $0-10$ mps & Mobility of nodes \\
\hline 6 & No. of Connection & 8 & Connections \\
\hline 7 & Mobility Model & Random & Mobility direction \\
\hline 8 & Routing Protocols & AODV, DSR & Path-finding \\
\hline 9 & MAC Protocol & $\begin{array}{c}802.11 D C F, \\
802.11 M A C\end{array}$ & Wireless Protocol \\
\hline
\end{tabular}


Simulations have been carried out using QualNet5.0, to determine the impact of density of nodes on the performance of two MANET routing protocols i.e. AODV and DSR using Random Waypoint Mobility Model and the simulation parameters are shown in Table I.

The following performance parameters are compared for AODV and DSR routing protocols:

Packet Delivery Ratio (PDR) is the ratio of data packets delivered successfully to destination nodes and the total number of data packets generated for those destinations. It is characterized by the packet loss rate, which limits the throughput of the network. For better performance of the routing protocol, the delivery ratio should be higher. PDR is determined as:

$$
P D R=\left(P_{r} / P_{s}\right) \times 10
$$

where $P_{r}$ is the total packets received and $P_{s}$ is the total packets sent. Fig. 3 shows the fraction of the originated application data packets which each protocol was able to deliver, as a function of nodes. The packet delivery ratio increases as the number of nodes decreases as there is less congestion in the network. Both the protocols i.e. AODV and DSR have maximum delivering of 6.5 and 22.6667 of the packets, respectively against the transmission of 24 packets of data. From these results, a simple conclusion has been made that DSR has a higher packet delivery ratio $(90.6668 \%)$ than that of AODV (26\%).

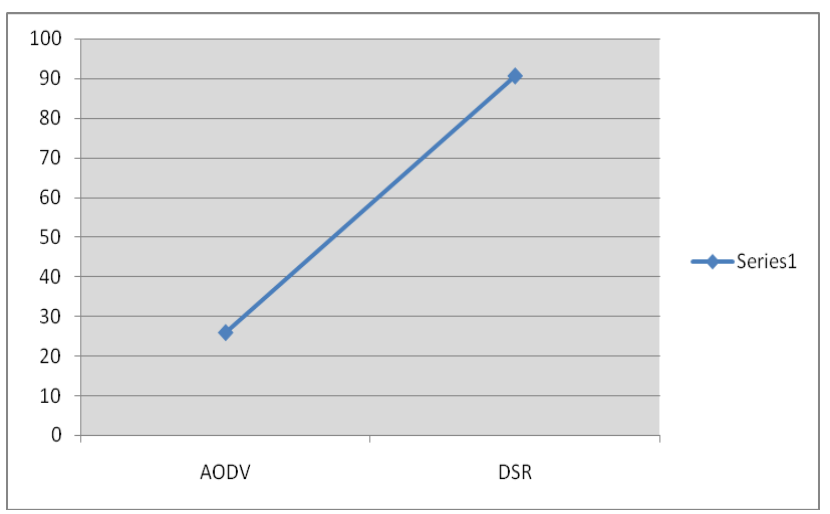

Fig. 3. Packet delivery ratio.

Average End to End Delay $\left(D_{\text {avg }}\right)$ is the time taken for a packet to travel from the source node application layer of the destination node. It also includes the route discovery wait time that may be experienced by a node when a route is initially not available. The average end to end delay is determined as:

$$
D_{\text {avg }}=\Sigma\left(t_{r}-t_{s}\right) / P_{r}
$$

where $t_{s}$ is the packet send time and $t_{r}$ is the packet receive time for the same packet at destination node. The average delay for these two routing protocols as shown in Fig. 4. It has been shown that DSR has minimum delay of 0.741488 sec. When requesting a new route, DSR first searches the route cache storing routes information it has learned over the past routing discovery stage and has not used the timer threshold to restrict the stale information which may lead to a routing failure.

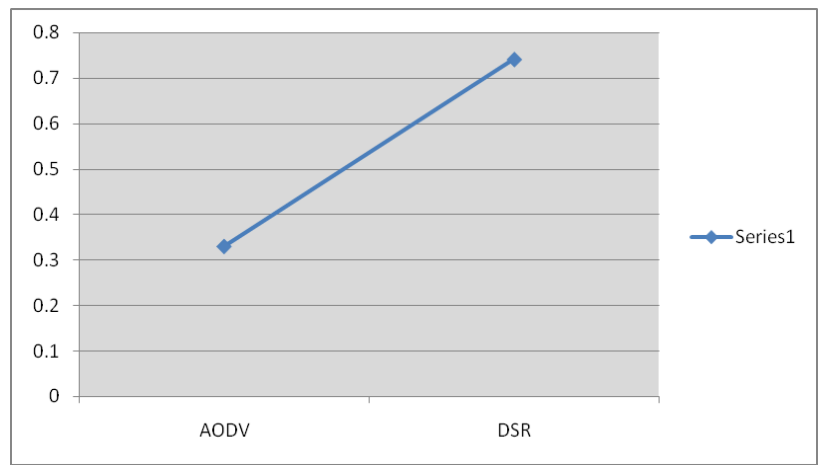

Fig. 4. Average end to end delay.

Reference [13] Moreover, DSR needs to put the route information not only in the route reply message but also in the data packets which relatively make the data packets longer than before. Both of the two mechanisms make DSR to have a long delay than other protocols. On the other hand, AODV has minimum delay i.e. $0.32961 \mathrm{sec}$.

Throughput: It is the average rate of successful message delivery over a communication channel. The average end to end throughput is shown in Fig. 5 which reflects the usage degree of the network resources for the typical routing protocols. With an offered load of 1 packets/sec, the maximum throughput is approximately $6 \mathrm{kbps}$. The throughput increases quickly for DSR which is 3659.On the other hand, AODV has throughput 1537.5 which is less in comparison to DSR.

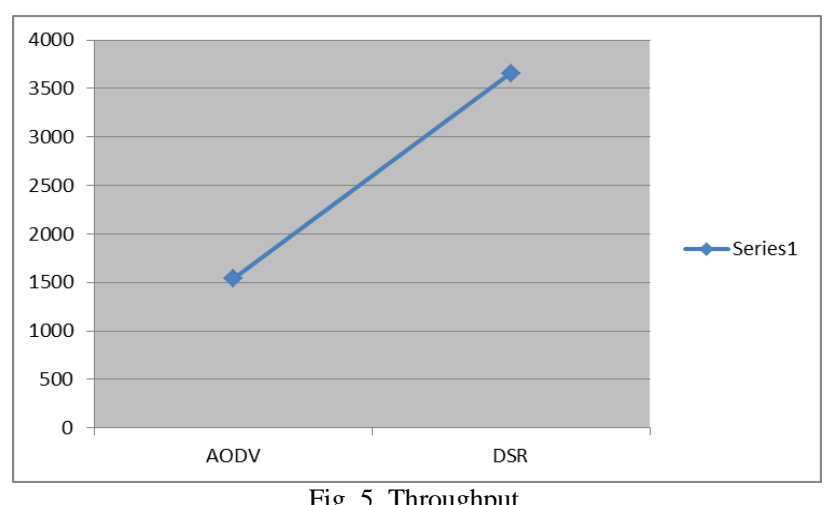

Average Jitter Value: It signifies the packets from the source will reach the destination with different delays. A packet's delay varies with its position in the queries of the routers along the path between source and destination as shown in Fig. 6. After implementation, AODV and DSR have $0.161342 \mathrm{sec}$. and $0.171953 \mathrm{sec}$. values respectively.

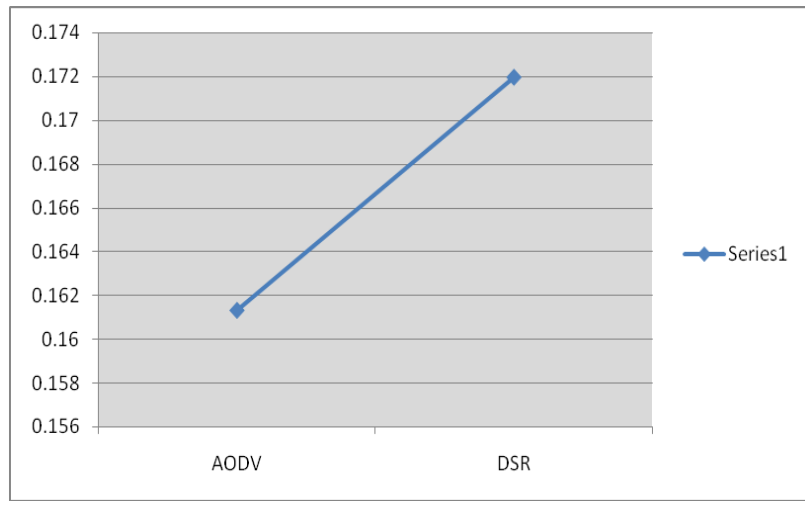

Fig. 6. Average jitter value. 
Average Packet Loss: This is the number of packets lost due to incorrect or unavailable routes and MAC layer collisions. Fig. 7 shows the relationship between the network size and the average packet dropped of the typical protocols which indicates the reliable degree of each protocol. After implementation, it is observed that AODV and DSR have the same performance in terms of average packet loss of 1.3.

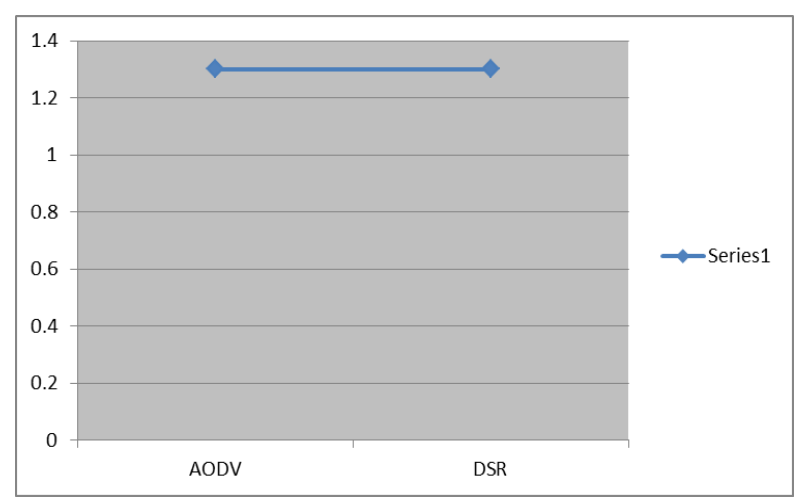

Fig. 7. Average packet loss.

\section{Discussions}

All the protocols have been simulated using the same parameters as discussed in Table I to ensure the comparison of all performance parameters as mentioned above. From the observation, the evaluation of QoS performances for two MANET protocols: AODV and DSR have been discussed. The analysis has been done through simulation using commercial and highly reliable QaulNet5.0 simulator. These performances metrics considered are the packet delivery ratio, average end to end delay, throughput, average jitter value and packet loss. It is observed that an increase in node density has different impact on all routing protocols under various mobility patterns, i.e. a degradation of the network performance. However, the degree of degradation varies for different combinations of protocols. The performance of RWP model provides a baseline to judge the quality of routing protocols when there is no group movement. Table II summarizes the performances comparison of two routing protocols for mobile ad hoc networks. " 1 " denotes for the best performance while " 2 " for the poor performance.

TABLE II: PERFORMANCE COMPARISON WITH 50 NODES

\begin{tabular}{|c|c|c|}
\hline \multirow{2}{*}{ Metrics } & \multicolumn{2}{|c|}{ Protocols } \\
\cline { 2 - 3 } & AODV & DSR \\
\hline Packet Delivery Ratio & 2 & 1 \\
\hline Average end to end delay & 1 & 2 \\
\hline Throughput & 2 & 2 \\
\hline Average jitter & 1 & 1 \\
\hline Packet Loss & 1 & \\
\hline
\end{tabular}

\section{CONCLUSIONS}

In this paper, the behaviour of MANET routing protocols under Random Waypoint Model has been analyzed. The results of our extensive QualNet5.0 simulations clearly indicate the significant impact of simulation parameters has on routing performance. We had taken the protocol as best and worst in respect of all major performance metrics. DSR provides better performance than AODV in throughput and packet delivery ratio metrics and poor performance for end to end delay and average jitter, whereas packet loss has same dropping in both protocols. The work done in this research aims to develop an understanding of the effects of mobility pattern on routing performance.

\section{REFERENCES}

[1] A. Maurya, D. Singh, A. Kumar, and R. Maurya, "Random waypoint mobility model based performance estimation of on-demand routing protocols in MANET for CBR applications," in Proc. International Conference on Computing for Sustainable Global Development (INDIACom), 2014, pp. 835-839.

[2] L. Khan, F. Khan, N. Khan, M. Khan, and P. Bilal, "Effect of network density on the performance of MANET routing protocols," in Proc. International Conference on Circuits, Power and Computing Technologies (ICCPCT), 2013, pp. 1089-1092.

[3] S. Biradar, H. Sarma, K. Sharma, S. Sarkar, and C. Puttamadappa, "Performance comparison of reactive routing protocols of MANETs using group mobility model," in Proc. International Conference on Signal Processing Systems, 2009, pp. 192-195.

[4] N. Adam, M. Ismail, and J. Abdullah, "Effect of node density on performances of three MANET routing protocols," in Proc. International Conference on Electronic Devices, Systems and Applications (ICEDSA), 2010, pp. 321-325.

[5] N. Sakar and W. Lol, "A study of MANET routing protocols: Joint node density, packet length and mobility," in Proc. IEEE Symposium on Computers and Communications (ISCC), 2010, pp. 515-520.

[6] S. Hosseini and H. Farrokhi, "The impacts of network size on the performance of routing protocols in mobile Ad-Hoc networks," in Proc. Second Pacific-Asia Conference on Circuits, Communications and System (PACCS), 2010, pp. 18-22.

[7] K. Amjad and A. Stocker, "Impact of node density and mobility on the performance of AODV and DSR in MANETs," in Proc. 7th International Symposium on Communication Systems Networks and Digital Signal Processing (CSNDSP), 2010, pp. 61-65.

[8] F. Maan and N. Mazhar, "MANET routing protocols vs mobility models: A performance evaluation," in Proc. Third International Conference on Ubiquitous and Future Networks (ICUFN), 2011, pp. 179-184.

[9] G. Vijayavani and G. Prema, "Performance comparison of MANET routing protocols with mobility model derived based on realistic mobility pattern of mobile nodes," in Proc. IEEE International Conference on Advanced Communication Control and Computing Technologies (ICACCCT), 2012, pp. 32-36.

[10] U. Bhatt, A. Dangarh, A. Kashyap, and A. Vyas, "Performance analysis of AODV \& DSR routing protocols for MANET," in Proc. Fourth International Conference on Communication Systems and Network Technologies (CSNT), 2014, pp. 254-258.

[11] L. T. Yi, Y. J. Zhai, Y. Wang, J. Yuan, and Y. Ilsun, "Impacts of internal network contexts on performance of MANET routing protocols: A case study," in Proc. Sixth International Conference on Innovative Mobile and Internet Services in Ubiquitous Computing (IMIS), 2012, pp. 231-236.

[12] A. Al-Hemyari, R. Hassan, M. Ismail, and S. Saeed, "Performance evaluation of node mobility and varying group density on long lifetime multicast routing protocol in MANETs," in Proc. IEEE Malaysia International Conference on Communications (MICC), 2013, pp. 271-276.

[13] B. Gouda, A. Mandai, and K. Narayana, "Simulation and comparative analysis of energy conservation performance metric for ERAODV, AODV and DSDV routing protocols in MANET," in Proc. World Congress on Information and Communication Technologies (WICT), 2012, pp. 278-292.

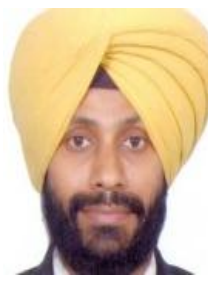

Jagpal S. Ubhi received the B.E. degree and M.S degree from Thapar Institute of Engineering \& Technology, Patiala (Punjab) in 1994 and 2000 respectively. He received his Ph.D. degree from Punjab Technical University, Jalandhar in 2011. He is now working as an associate professor at the Sant Longowal Institue of Engineering and Technology, Longowal, India. He is in teaching profession for the last 20 years. His current research interests include 
digital communication theory, wireless fading channels, diversity systems, wireless digital communications and VLSI design.

Dr. Ubhi was awarded 'Certificate of Merit' by Institution of Engineers (I) for his research paper published in the Journal of Electronics \& Telecom Engineering Division of the Institution during 2006-2007. He chaired a session in the International Conference of Signal and Image Engineering under the aegis of WCE held during 6-8 July, 2011 which was organized by IAENG at the South Kensington Campus, Imperial College, London, UK. $\mathrm{He}$ is also life member of IE(India), IETE(India), IAENG.

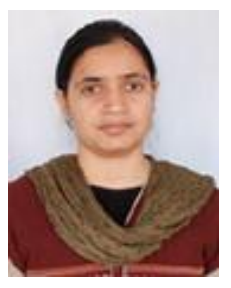

Satveer Kaur has received her bachelor of technology degree in information technology from the Technological Institute of Textile \& Science, Bhiwani, Haryana, India in 2006. She has done her master of technology degree in computer science and engineering from Chaudhary Devi Lal University, Sirsa, Haryana, India in 2009 and currently pursuing her Ph.D. degree at SLIET, Longowal (India). She is currently working as an assistant professor in CSE Department of Guru Nanak Dev University Regional Campus, Sathiala, Distt. Amritsar since 2012. Her research interests include wireless networks, mobile Ad-Hoc networks, and vehicular Ad-Hoc network. 